\title{
Defining and refining sperm selection strategies
}

\author{
David F. Albertini ${ }^{1}$
}

Published online: 26 May 2016

(C) Springer Science+Business Media New York 2016

Misura ciò che è misurabile, e rendi misurabile ciò che non lo è

(Measure what can be measured, and make measurable what cannot be measured)

\section{- Galileo Galilei}

After tracking some of the remarkable advances in imaging embryos in previous months, JARG turns attention to the male gamete this month keeping in mind the essence of the practice of human ARTs: selecting the very best of oocytes, sperm, and embryos from an admixture of perfection and imperfection. What makes a given sperm identifiable as a "lead candidate" for ICSI, or the population behavior from a fresh or frozen ejaculate destined for a fresh IVF cycle, is largely left in the hands of the resident andrologist following the latest WHO guidelines.

Most of what we can measure in the process of selection carries with it our tendencies to delineate those properties or activities having to do with what mature sperm would engage in during the erstwhile process of fertilization, somewhere within the distal reaches of the Fallopian tube. The microenvironment fully capacitated sperm find themselves in must be one of turbulence, signaled by the arrival of a hormone-laced expanded cumulus mass being fondled by ciliary activity and buoyed by secretions of the oviductal epithelium.

It is no wonder then that our perceptions of what it takes to effect fertilization would rest in the highest degree of motility and normal morphology to distinguish better sperm from the

Capsule As our knowledge base expands on the properties of the male gamete that contribute directly to the success or failure of early human development, the need to redefine and refine our assays for sperm quality presents new challenges to the future of human ARTs.

David F. Albertini

DALBERTINI@kumc.edu

1 University of Kansas Medical Center, Kansas, KS, USA stragglers and misfits that have joined along in the semen preparation now gracing the embryology lab for ICSI or conventional IVF; of course, neither of these assignments even remotely resemble the natural microenvironment alluded to above. But, whether by machine intervention or traditional semen analysis, the chosen few will have been selected based on these properties (see Volpes and colleagues, The pellet swim-up is the best technique for sperm preparation during in vitro fertilization procedures, 10.1007/s10815-016-0696-2).

For the chosen ones, what we discuss above is only the end of a long journey for the spermatozoon. As has become more apparent, beginning with their differentiation in the testis, and extending through their storage and exposure to seminal secretions, these cellular sentinels of paternity are acquiring baggage that will play a central role in assuring that the full paternal legacy is delivered in a form that will be utilized by the nascent zygote- remember the exosomes, small RNAs that form the substance of their cargo from recent issues of JARG.

Developing new measurement tools dictates having defined physical, chemical, or molecular properties suspected of playing a role consistent with optimizing gametic contributions to the ART process. That sperm maturation in the epididymis includes a variety of alterations is well known, and one of the first to be appreciated was the change in plasma membrane net negative charge due to the acquisition of sialic acid residues. This month, Simon and colleagues from the University of Utah report on refinement of a microelectrophoresis technique designed to detect and separate sperm based on cell surface properties previously shown by this group to provide improvements in embryo developmental potential (Optimization of microelectrophoresis to select highly negatively charged sperm, 10.1007/s10815-016-0700-x). The work stands out as an example of the measured progress resulting from collaborative basic and applied science, illustrates the importance of using multiparametric outputs 
when introducing a new assay, and notes that any application to clinical selection of non-apoptotic sperm will have to await rigorous experimentation to carry such studies beyond the proof-of-principle stage.

Animal models continue to provide new insights into the process of ICSI as shown by Ansalone and Iuso and their collaborators from the University of Teramo (Plasma membrane and acrosome loss before ICSI is required for sheep embryonic development, 10.1007/s10815-016-0709-1). In particular, their studies revisit the question of what properties of sperm utilized during ICSI produce the best developmental outcomes, emphasizing the relative contributions of plasma membrane removal in addition to the acrosome reaction in achieving improved clinical outcomes.

And finally, one of the long-standing principles of fertilization in mammals has rested on definition of the role of PLC $\zeta$ in egg activation, raising for many the possibility that cases of failed fertilization may in the end be attributable to genetic defects or pathophysiological alterations in sperm.
This viewpoint is challenged by the paper from the group of Vassena from Barcelona (PLC $\zeta$ sequence, protein levels, and distribution in human sperm do not correlate with semen characteristics and fertilization rates after ICSI, 10.1007/s10815016-0718-0). While discourse continues over the potential link between PLC $\zeta$ and its role in egg activation, it should come as no surprise to the JARG readership that once again, widely acknowledged hallmarks of gamete physiology are being labeled as insufficient to explain deficits in ART despite their previous implication in processes now reaching new levels of complexity.

In conclusion, if we chose to abide by Galileo's advice, we should be prepared to accept that many of the measurables when it comes to the assessment of gamete quality will take on the character of constructions that best align with our biases, which for better or worse, tentatively serve the needs of an ever-changing and evolving platform of human ARTs. We invite our readership to extend this notion to the field of preimplantation genetic diagnosis (PGS), the focus for our next issue of JARG. 